Thomas Meyer $\cdot$ Rüdiger Ontrup $\cdot$ Christian Schicha

Die Inszenierung des Politischen 
Thomas Meyer $\cdot$ Rüdiger Ontrup

Christian Schicha

Wissenschaftliche Redaktion Carsten Brosda

\title{
Die Inszenierung des Politischen
}

\author{
Zur Theatralität \\ von Mediendiskursen
}

Springer Fachmedien Wiesbaden GmbH 
Die Deutsche Bibliothek - CIP-Einheitsaufnahme

Alle Rechte vorbehalțen

(1) Springer Fachmedien Wiesbaden 2000

Ursprünglich erschienen bei Westdeutscher Verlag $\mathrm{GmbH}$, Wiesbaden, 2000

Lektorat: Hildegund Stapf, Universität Mainz

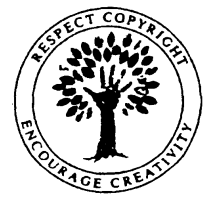

Das Werk einschließlich aller seiner Teile ist urheberrechtlich geschützt. Jede Verwertung außerhalb der engen Grenzen des Urheberrechtsgesetzes ist ohne Zustimmung des Verlags unzulässig und strafbar. Das gilt insbesondere für Vervielfältigungen, Übersetzungen, Mikroverfilmungen und die Einspeicherung und Verarbeitung in elektronischen Systemen.

www.westdeutschervlg.de

Höchste inhaltliche und technische Qualität unserer Produkte ist unser Ziel. Bei der Produktion und Verbreitung unserer Bücher wollen wir die Umwelt schonen: Dieses Buch ist auf säurefreiem und chlorfrei gebleichtem Papier gedruckt. Die Einschweißfolie besteht aus Polyäthylen und damit aus organischen Grundstoffen, die weder bei der Herstellung noch bei der Verbrennung Schadstoffe freisetzen.

Umschlaggestaltung: Horst Dieter Bürkle, Darmstadt 


\section{Inhaltsverzeichnis}

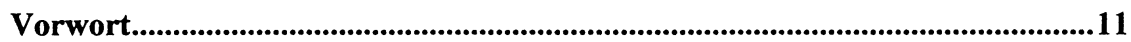

1 Einleitung .................................................................................................................. 13

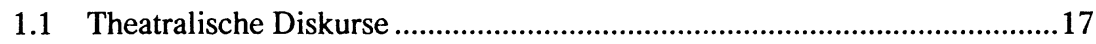

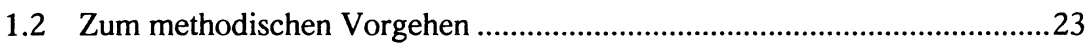

1.3 Zusammenfassung der Ergebnisse .........................................................27

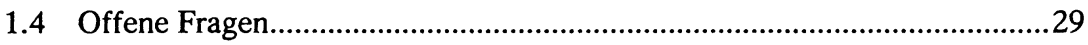

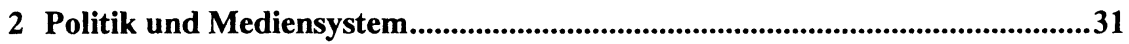

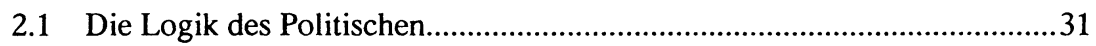

2.2 Systemgrenze zwischen Mediensystem und politischem System ................36

2.3 Verschwinden des Politischen im Supersystem?...........................................39

3 Theatralität in der Mediengesellschaft Aspekte des Theatralitätskonzepts......................................................................45

3.1 Geschichte und Funktion der Theatermetapher........................................47

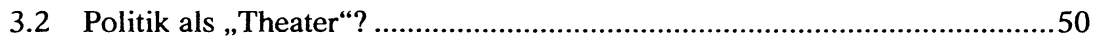

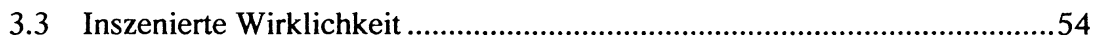

3.3.1 Wirkungsorientierung und Selbstreflexivität ....................................58

3.3.2 Das Zusammenspiel unterschiedlicher Medien
und Zeichensysteme

3.3.3 Prozessualität ............................................................................62

3.4 Theatralisierung....................................................................................62

3.4.1 Transformationsprozesse im Zeichengebrauch

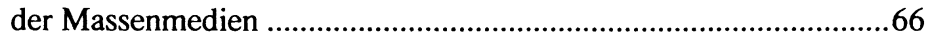

3.4.2 Die Theatralisierung des Publikums ................................................70

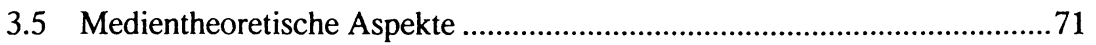




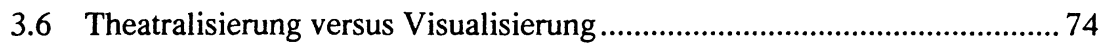

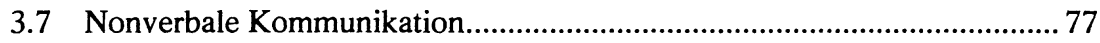

3.7.1 Zum Verhältnis verbaler und nonverbaler Äußerungen .................80 80

3.7.2 Politische Diskussionen im Fernsehen ....................................... 85

3.8 Inszenierungspotentiale ........................................................... 90

3.9 Von der Inszenierung zum Test ....................................................... 91

4 Rhetorik und normative Theorie ..............................................................95

4.1 Maßstäbe massenmedialer Kommunikation ..........................................98

4.1.1 Grundlagen der Kommunikationspolitik (BVerfGE) ....................99

4.1.2 Information ........................................................................ 103

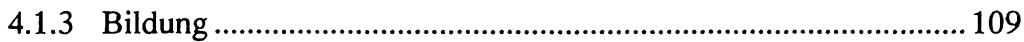

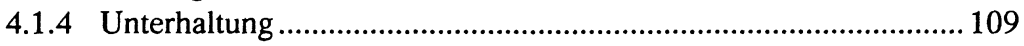

4.2 Die Diskurstheorie als normativer Rahmen ........................................ 111

4.2.1 Diskurs und Argumentation................................................. 112

4.2.2 Diskursregeln............................................................... 115

4.3 Modelle der Diskurstheorie................................................................ 116

4.3.1 Jürgen Habermas ................................................................... 117

4.3.2 Richard Münch ................................................................... 122

4.3.3 Fazit ............................................................................. 124

4.4 Medienrhetorik und Medieninszenierung ....................................... 126

4.4.1 Medieninszenierung........................................................ 126

4.4.2 Rhetorische Kommunikation ............................................. 128

4.4.3 Medienrhetorik ................................................................... 131

4.4.4 Die ,Macht“ der Bilder........................................................... 132

4.4.5 Visuelle Politikdarstellung...................................................... 134

4.4.6 Glaubwürdigkeit .................................................................. 135

4.4.7 Symbolische Politik .............................................................. 136

4.4.8 Infotainment ................................................................... 138

4.5 Rhetorik und Theatralität - Ein Zwischenfazit ................................. 141

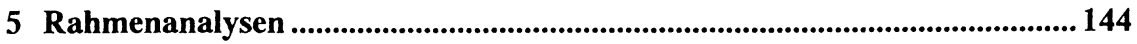

5.1 Politisches Magazin: „Frontal“ (ZDF) ............................................ 144

5.1.1 Allgemeine Merkmale politischer Magazine............................. 144

5.1.2 Das Politikmagazin „Frontal“ (ZDF) ....................................... 146

5.2 Politisches Magazin: „Kontraste“ (ARD) ........................................... 152 
5.3 Politisches Magazin: „Spiegel-TV“ (RTL) .........................................154

5.4 Politisches Magazin: „ZAK“ (ARD) ..................................................156

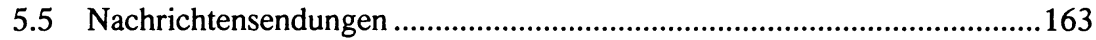

5.5.1 Die Entwicklung des dualen Systems .......................................164

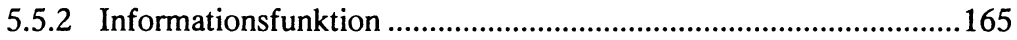

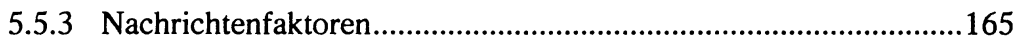

5.5.4 Symbolische Politikinszenierung ..............................................166

5.5.5 Inhalte der Fernsehnachrichten ...............................................168

5.5.6 Visualisierung/Optische Aufmachung ........................................169

5.5.7 Personalisierung...................................................................170

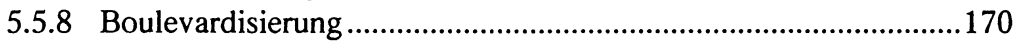

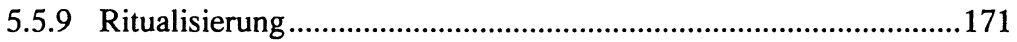

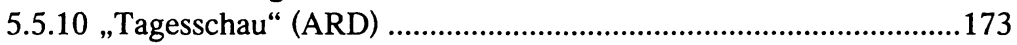

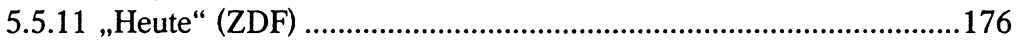

5.5.12 Nachrichten der kommerziellen Betreiber ................................177

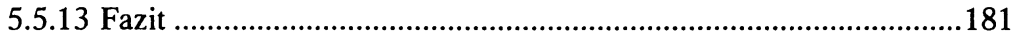

5.6 Nachrichtenjournal: „Tagesthemen“ (ARD) ..........................................182

5.7 Politischer Talk: „Presseclub“ (ARD) ..................................................187

5.8 Politischer Talk: „Talk im Turm“ (SAT 1) ..........................................190

6 Strategien medialer Inszenierungen -

Typenbeispiele aus der Stichtagsanalyse ..............................................194

6.1 Strategien der Personalisierung ........................................................... 198

6.1.1 Personalisierende Strategie ........................................................199

6.1.2 Personale Strategie ..............................................................201

6.2 Reine Unterhaltungsdramaturgie...................................................202

Fallbeispiel: „Frontal“, ZDF, 4. Juni 1996,

Thema: Dumme Verbrecher ............................................................203

6.3 Politische Satire ...............................................................................204

Fallbeispiel: „ZAK“, ARD, 2. Juni 1996,

Thema: Öffentliches Rekrutengelöbnis ............................................204

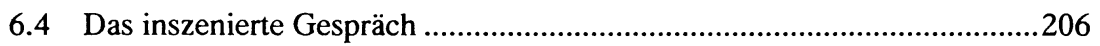

6.4.1 Das Streitgespräch...............................................................207

Fallbeispiel: „Talk im Turm“, SAT 1, 2. Juni 1996 .............................209

6.4.2 Das konfrontative Gespräch..................................................2220

Fallbeispiel: „ZAK“-Interview mit Wendelin Wiedeking,

ARD, 2. Juni 1996 


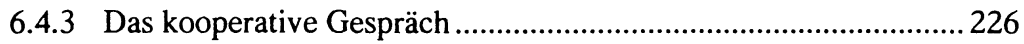

Fallbeispiel: „Presseclub“, ARD, 2. Juni 1996 .......................................... 227

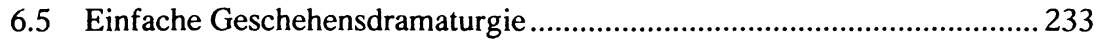

Fallbeispiel: „Tagesschau“, ARD, 2. Juni 1996,

Thema: Vorbereitungen Nato-Konferenz .............................................. 233

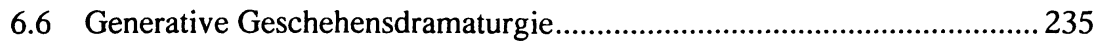

Fallbeispiel: „Tagesthemen“, ARD, 2. Juni 1996, Thema: Israel ............. 236

6.7 Formalästhetisch narrative Geschehensdramaturgie ................................241

Fallbeispiel 1: „Frontal“, ZDF, 4. Juni 1996,

Thema: Regierungsumzug nach Berlin.................................................... 241

Fallbeispiel 2: „Kontraste“, ARD, 6. Juni 1996,

Thema: Regierungsumzug nach Berlin ................................................. 247

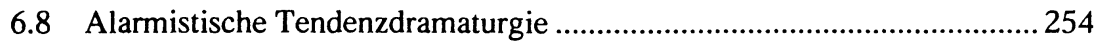

Fallbeispiel: „Spiegel TV Magazin“, RTL, 2. Juni 1996,

Thema: 'Die amerikanische Gefahr -

Deutschland auf dem Weg in die Mac-Job-Gesellschaft' .........................254

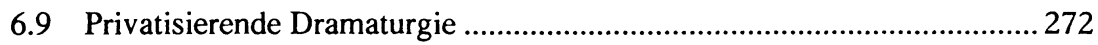

Fallbeispiel: „Spiegel TV“, RTL, 2. Juni 1996,

Thema: Geheimdienst im Wendland...................................................... 272

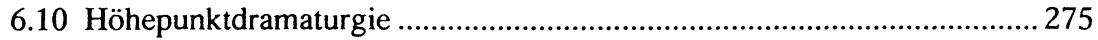

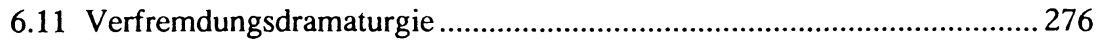

Fallbeispiel: „ZAK“, ARD, 2. Juni 1996,

Thema: Zwei Azubis teilen sich eine Lehrstelle ........................................ 277

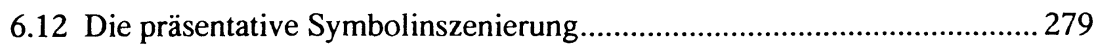

Fallbeispiel: „Spiegel-TV“, RTL, 2. Juni 1996,

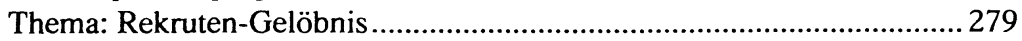

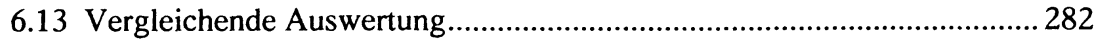

6.14 Zusammenfassung der empirischen Ergebnisse..................................... 285

6.14.1 Kategorien des Politischen ........................................................ 286

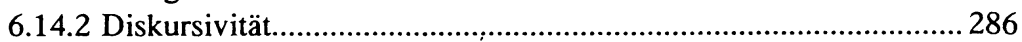

6.14.3 Verhältnis von Inszenierung und Argumentativität....................... 287

6.14.4 Inszenierung und Nutzungsentscheidung .....................................28

7 Visualisierungsstrategien............................................................................... 291

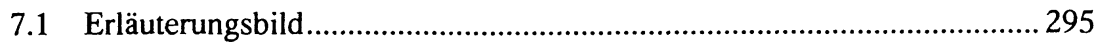

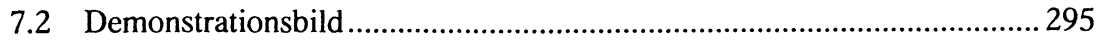




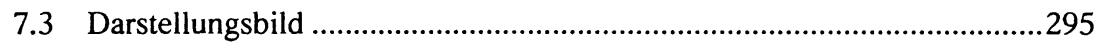

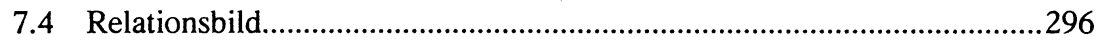

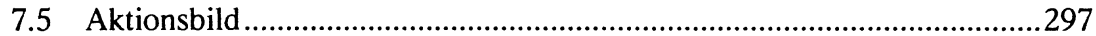

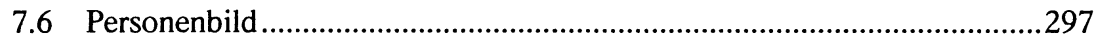

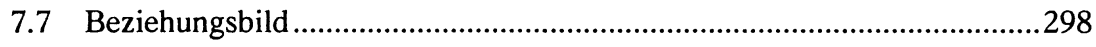

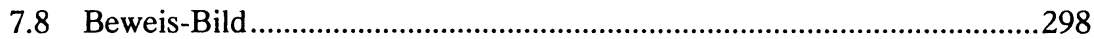

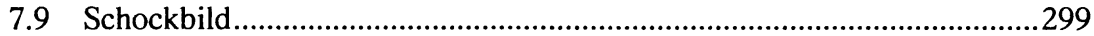

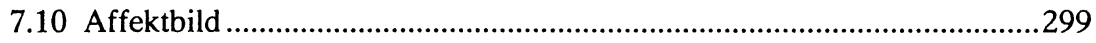

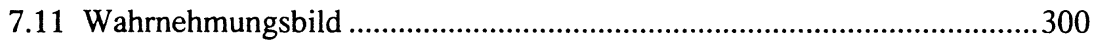

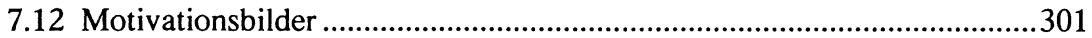

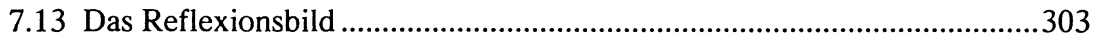

8 Fazit aus den empirischen Untersuchungen........................................306

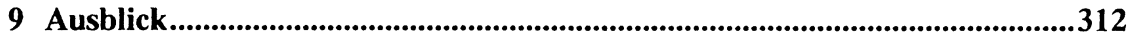

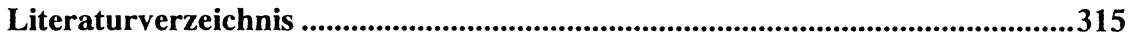




\section{Vorwort}

Die vorliegende Publikation dokumentiert die Ergebnisse eines Forschungsprojektes mit dem Titel „Argumentativität und Theatralität politischer Diskurse in der Mediengesellschaft", das an der Universität Dortmund im Fach Politikwissenschaft durchgeführt worden ist.

Die Arbeiten der Dortmunder Arbeitsgruppe liefern dabei einen Baustein zu dem von der Deutschen Forschungsgemeinschaft (DFG) gefördertem Schwerpunktprogramm „Theatralität - Theater als kulturelles Modell in den Kulturwissenschaften". Innerhalb dieses Programmes ist die so häufig erhobene Forderung nach einem interdisziplinären wissenschaftlichen Austausch. in einem bemerkenswert umfassenden und produktiven Sinn realisiert worden. Die intensiven wissenschaftlichen Kontakte, die sich im Rahmen der Jahrestreffen unter der Koordination von Prof. Dr. Erika Fischer-Lichte zwischen den Teilnehmern des Schwerpunktprogrammes an der FU Berlin ergeben haben und im Verlauf des Forschungsprojektes intensiviert werden konnten, gaben wertvolle Impulse für die Forschungsarbeit. Auf zusätzlichen thematischen Teilkolloquien an den Universitäten Duisburg und Dortmund sowie beim SIGMA-Institut in Mannheim ergaben sich darüber hinaus weiterführende Gelegenheiten zu einem Austausch über den Fortgang der jeweiligen Forschungsprojekte.

Durch die intensive interdisziplinäre Kooperation mit den Kollegen aus den Fachdisziplinen der Theaterwissenschaft, Soziologie, Philosophie, Psychologie und Sozialwissenschaft hat sich der Fokus des Forschungsgegenstandes maßgeblich erweitert. Einige zentrale Ergebnisse der daraus resultierenden Kooperation mit den Fachkollegen aus den unterschiedlichen Disziplinen sind in dem unlängst von Christian Schicha und Rüdiger Ontrup herausgegebenen Sammelband „Medieninszenierungen im Wandel - Interdisziplinäre Zugänge" dokumentiert, an dem zahlreiche Autoren des Schwerpunktprogramms mitgewirkt haben. Diese Publikation schließt an die Studien der Sammelbände „Inszenierungsgesellschaft“ (hrsg. von Herbert Willems und Martin Jurga) und „Medien im Wandel“ (hrsg. von Udo Göttlich, Jörg-Uwe Nieland und Heribert Schatz) an.

Unser besonderer Dank gilt einer Reihe von Personen, die den Verlauf unseres Projektes kritisch und konstruktiv begleitet haben: Eine große Hilfe waren uns die zahlreichen Fachgespräche der ebenfalls interdisziplinär ausgerichteten „Dortmunder Arbeitsgruppe“ mit den Soziologen Prof. Dr. Hartmut Neuendorff und Dr. Jörg Tykwer sowie den Journalisten Prof. Dr. Günter Rager und Dipl.-Journ. Lars Rinsdorf und unserem Kollegen PD Dr. Udo Vorholt vom Institut für Politikwissenschaft. Sie ermöglichten uns eine weitergehende Perspektivierung des Untersu- 
chungsgegenstandes, die über eine rein „politikwissenschaftliche“ Zentrierung deutlich hinausging. Die Arbeitsteilung mit den Kollegen vom Institut für Journalistik bei der Analyse der Fallbeispiele des Stichtagsmodells übertrug neben vielem anderen die aufwendigen quantitativen Analysen den Journalisten, während der politikwissenschaftliche Schwerpunkt auf der theoretischen Fundierung und der qualitativen Interpretation exemplarischer Fernsehanalysen lag. Durch das unterschiedliche methodische Vorgehen bei der Auswertung der Fallbeispiele konnten beide Gruppen jeweils von den Ergebnissen der Kollegen in ausschlaggebendem Ausmaß für die Gesamtuntersuchung profitieren.

Besonders hervorheben möchten wir die enge und fruchtbare Kooperation mit den Duisburger Kollegen Dr. Udo Göttlich und Dipl. Soz.-wiss. Jörg-Uwe Nieland unter der Leitung von Prof. Dr. Heribert Schatz sowie den Austausch mit dem Bamberger Psychologen Prof. Dr. Günter Laux und der Arbeitsgruppe unter der Leitung der Konstanzer Soziologen Prof. Dr. Hans- Georg Soeffner und PD Dr. Herbert Willems.

Wir bedanken uns zudem für die Kritik durch Prof. Dr. Dr. (USA) Peter Ludes und Prof. Dr. Ulrich Sarcinelli, deren wertvolle Anregungen wir gerne aufgegriffen haben.

Weiterhin möchten wir der Deutschen Forschungsgemeinschaft für die Finanzierung dieses Forschungsschwerpunktes und unseres Teilprojektes danken.

Unser besonderer Dank gilt abschließend unserem Kollegen Carsten Brosda, dem die Verantwortung für die wissenschaftliche Redaktion des vorliegenden Buches oblag.

Dortmund, im Oktober 1999

Prof. Dr. Thomas Meyer

Rüdiger Ontrup M.A.

Dr. Christian Schicha 